\title{
Velocity Field Measurements of the California Sea Lion Propulsive Stroke Using Bubble PIV
}

\author{
Gino Perrotta ${ }^{1}$, Frank E. Fish ${ }^{2}$ (D), Danielle S. Adams ${ }^{2}$, Ariel M. Leahy ${ }^{2}$, Abigal M. Downs ${ }^{2}$ \\ and Megan C. Leftwich ${ }^{3, *}$ \\ 1 The Johns Hopkins University Applied Physics Laboratory, Laurel, MD 20703, USA; gino.perrotta@jhuapl.edu \\ 2 Department of Biology, West Chester University, West Chester, PA 19383, USA; ffish@wcupa.edu (F.E.F.); \\ DA762671@wcupa.edu (D.S.A.); AL916349@wcupa.edu (A.M.L.); ad846650@wcupa.edu (A.M.D.) \\ 3 Department of Mechanical and Aerospace Engineering, George Washington University, \\ Washington, DC 20052, USA \\ * Correspondence: mleftwich@gwu.edu
}

Citation: Perrotta, G.; Fish, F.E.; Adams, D.S.; Leahy, A.M.; Downs, A.M.; Leftwich, M.C. Velocity Field Measurements of the California Sea Lion Propulsive Stroke Using Bubble PIV. Fluids 2022, 7,3. https:// doi.org/10.3390/fluids7010003 Academic Editors: Sean P. Colin and John H. Costello

Received: 2 November 2021 Accepted: 10 December 2021 Published: 22 December 2021

Publisher's Note: MDPI stays neutral with regard to jurisdictional claims in published maps and institutional affiliations.

Copyright: (C) 2021 by the authors. Licensee MDPI, Basel, Switzerland. This article is an open access article distributed under the terms and conditions of the Creative Commons Attribution (CC BY) license (https:// creativecommons.org/licenses/by/ $4.0 /)$.

\begin{abstract}
California sea lions are among the most agile of swimming mammals. Most marine mammals swim with their hind appendages—flippers or flukes, depending on the species-whereas sea lions use their foreflippers for propulsion and maneuvering. The sea lion's propulsive stroke generates thrust by forming a jet between the flippers and the body and by dragging a starting vortex along the suction side of the flipper. Prior experiments using robotic flippers have shown these mechanisms to be possible, but no flow measurements around live sea lions previously existed with which to compare. In this study, the flow structures around swimming sea lions were observed using an adaptation of particle imaging velocimetry. To accommodate the animals, it was necessary to use bubbles as seed particles and sunlight for illumination. Three trained adult California sea lions were guided to swim through an approximately planar sheet of bubbles in a total of 173 repetitions. The captured videos were used to calculate bubble velocities, which were processed to isolate and inspect the flow velocities caused by the swimming sea lion. The methodology will be discussed, and measured flow velocities will be presented.
\end{abstract}

Keywords: marine mammal; California sea lion; velocity fields; bubble PIV; thrust production

\section{Introduction}

In situ flow measurements of swimming animals present significant technical and logistical challenges. Despite this, a rich body of literature exists for measuring both the locomotion and kinematics of swimming fish and the flow that they generate. Early successes often adapted digital particle image velocimetry (PIV) techniques to laboratory environments studying swimming animals. This enabled the wakes of a variety of steadily swimming animals to be measured while swimming in water channels [1-4] or freely swimming in stationary tanks $[5,6]$. Although most of these studies present two-dimensional data, a few have recently extended these techniques to collect volumetric flow fields around swimming fish $[7,8]$. Notably, this is in no way an exhaustive overview of laboratorybased flow measurements of swimming fish; we refer the interested reader to Triantafyllou (2000) [9] and, more recently, $\mathrm{Wu}$ (2011) [10], Lauder (2015) [11], and Costello 2020 [12].

The studies briefly mentioned above provide extensive insight into the mechanisms of unsteady propulsion, particularly for body/caudal fin-type swimming. However, they are laboratory studies; the animals' environments have been altered. Additionally, some animals cannot be safely studied in a laboratory setting. To combat this, a handful of in situ techniques have been developed to measure the flow field of animals swimming in their natural environments. Notably, the Self-Contained Underwater Velocimetry Apparatus (SCUVA) device [13], as well as its precursor studies [14], tracked particles naturally occurring in the water with a camera and laser setup operated by a freely swimming human 
diver. A later device extended the measurements to three dimensions [15]. These techniques, although impressive tools for studying the hydrodynamics of animals undisturbed by artificial laboratory settings, have their own limitations. The field of view is relatively small, and they are limited to animals found in the near-shore region (although work by Katija et al. has allowed for measurements of the flow around animals in deeper water using offshore remote-operated vehicles [16]).

To combat these limitations, researchers have developed robotic models to study the hydrodynamics of animals that cannot be studied in situ. In some cases, the entire animal-system is modeled [17-20], and in others, propulsive elements are isolated for systematic investigation [21-23]. Robotic models provide a platform to explore propulsion beyond what nature provides. The geometry and kinematics can be altered in ways that are impossible in animal studies. However, they lack the complexity and reality of in situ measurements. Thus, Fish et al. adapted laboratory PIV for safe use on larger animals [24,25]. As opposed to seeding the entire flow with reflective particles and illuminating a plane of the flow with a laser, they produced a sheet of small bubbles and used sunlight for global illumination. Then, underwater cameras imaged the flow in the same method as traditional PIV. A specific version of this, coined Bubble Image Velocimetry (BIV), was used by Ryu et al. [26]. However, Fish's method was more similar to traditional PIV and was used to measure the flow around a swimming dolphin and estimate the thrust produced during a tail stand maneuver [24,25]. In the current work, we will extend the modification of PIV of Fish et al. to measure the flow produced by a swimming sea lion, also using bubbles as seeding particles and sunlight as illumination. This is the first time that the flowfield of a swimming sea lion has directly been measured. We will present an extensive extension to the image processing required to measure flow under field conditions. Finally, we will present the flow generated by the propulsive stroke of an adult female sea lion freely swimming through a pool of stationary water.

\section{Materials and Methods}

\subsection{Data Collection}

All data were collected at the Science Learning and Exploration With the Help of Sea Lions (SLEWTHS) facility in Moss Landing, California. Three sea lions-Cali, Ariel, and Nemo-were trained to swim across a pool to a target. The target was positioned so that the sea lions would reach it by performing a single thrust-producing stroke-referred to as a 'clap' [27] - and so that the clap would occur within a measurement region. Sea lions produce thrust in a markedly different way from most high-performing swimmers. As opposed to producing thrust with their tails via some form of body/caudal fin locomotion $(\mathrm{BCF})$, the sea lion uses its large foreflippers to produce forward motion. The flippers are extended away from the body and then brought downward into the belly (the 'clap' mentioned above. This thrust-producing stroke was first described by Godfrey [28] and Feldkamp [29]. This process is outlined in Figure 1, panels a-c, and the large propulsive surface, the foreflipper, is shown in Figure 1d.

A perforated hose was placed at the bottom of the pool and connected to a tank of compressed air (Figure 2a). Traditional 2D PIV uses global seeding (with small reflective spheres that reliably follow the flow) and a sheet of light (typically, a laser beam which is manipulated with optics into a sheet of light). In this case, the opposite was true: sunlight globally illuminated the entire pool, but only a relatively thin sheet of the flow was seeded with particles. 

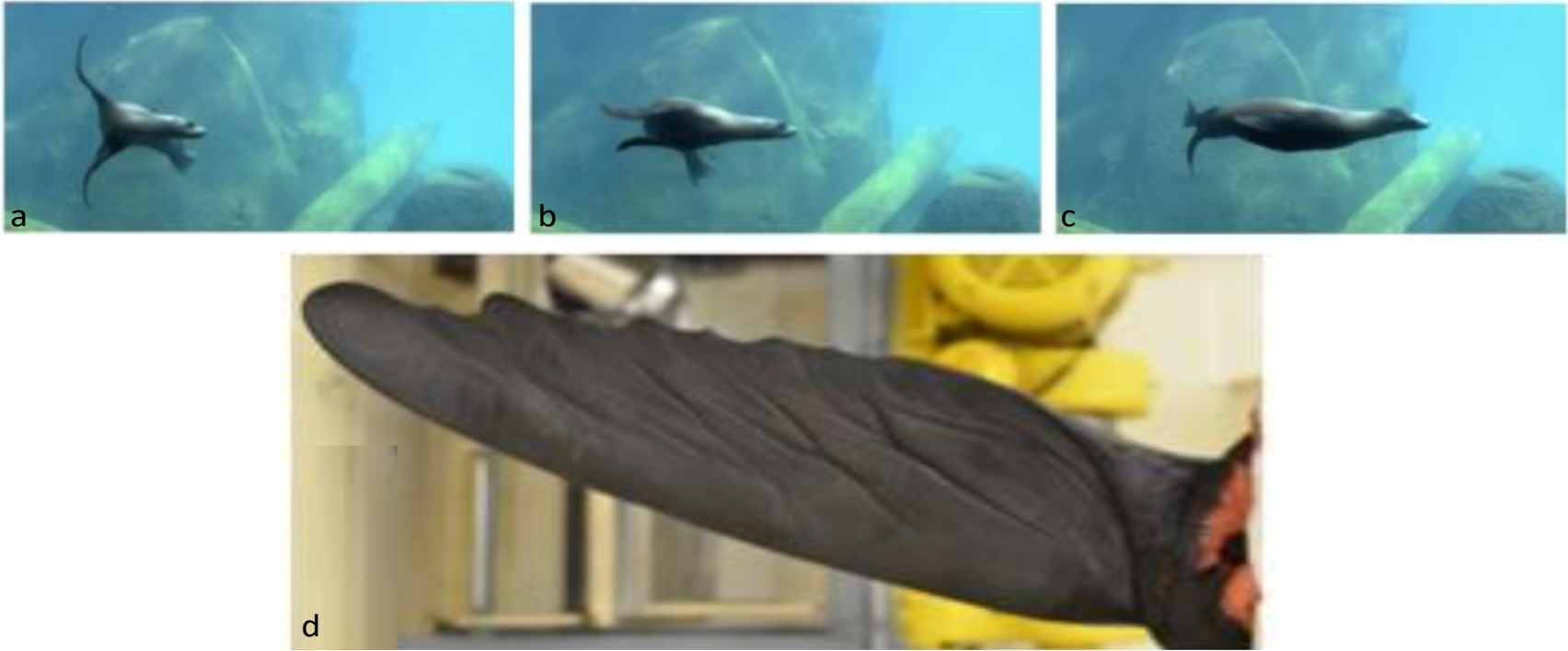

Figure 1. The top row (a-c) show the cycle of the sea lion's propulsive stroke. Thrust is produced by the large foreflippers. The bottom panel (d) is an image of a foreflipper of an adult female California sea lion. The image shows the underside of the flipper; the side that would touch the body in panel (c).
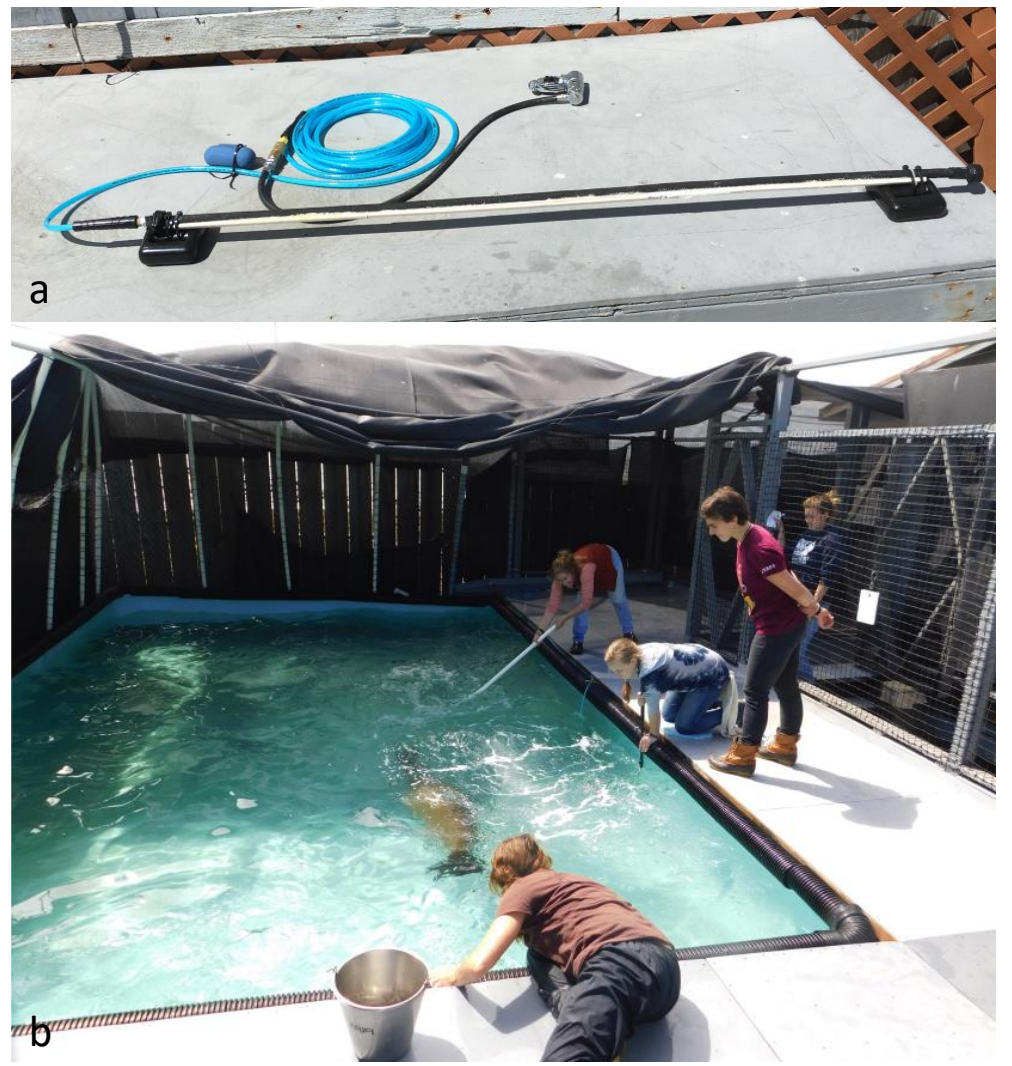

\section{Overhead Schematic}

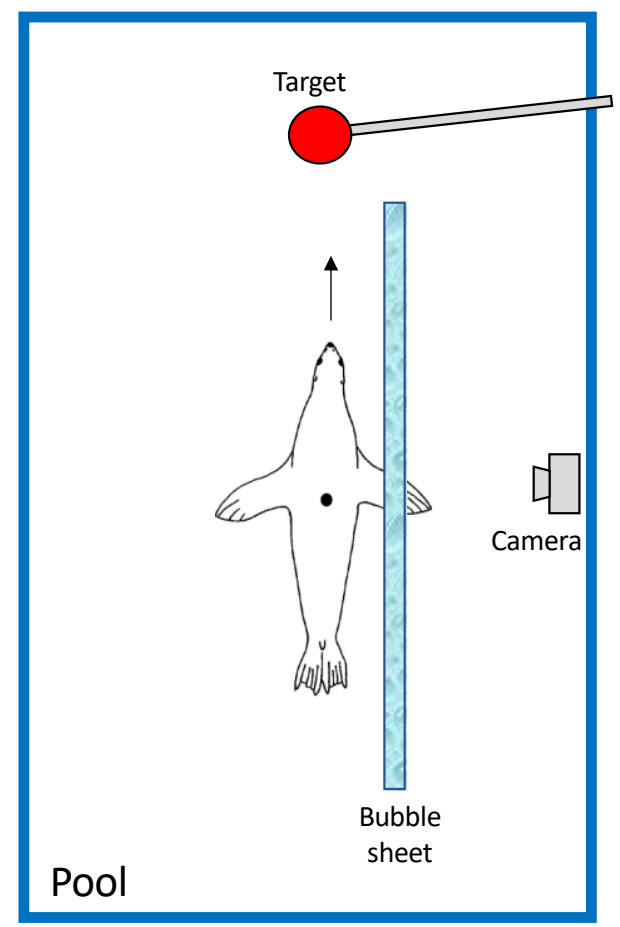

C

Figure 2. The top-left panel (a) shows the perforated hose used to generate a sheet of seeding bubbles. The bottom-left panel (b) is the pool used for data collection. The image includes SLEWTHS and research team members as well as Cali, the California sea lion that generated the present data. The left panel (c) is an overhead schematic of the experimental setup. The sea lion's swimming direction and locations of the bubble sheet and camera are shown.

In both cases, a camera was oriented at $90^{\circ}$ to the sheet (of light or, in this case, particles). In this study, images were taken with a GoPro HERO6 (GoPro, San Mateo, CA, 
USA) at 120 frames per second. The 1920-by-1080-pixel frame spanned 1.6-by-0.9 $\mathrm{m}$ at the measurement plane, providing a spatial resolution of $0.83 \mathrm{~mm}$ per pixel. Video captured by the GoPro camera was distorted around the edges by the high-field-of-view lens. This was apparent during length scale calibration; distance per pixel was higher at the border of the frame than at the center.

To preserve image clarity in the more relevant center region, the videos were not de-warped. Instead, the camera was arranged to view a larger area than was needed, and the distorted borders of the resulting measurements were discarded. Figure $2 b$ shows the SLEWTHS and research team members during data collection, and Figure $2 \mathrm{c}$ shows an overhead schematic of the experimental setup. In total, 173 raw data runs were collected, which produced many misses and 36 measurements of the desired event (processed data from all 36 successful runs are available upon request).

\subsection{Image Processing}

\subsubsection{Image Channel Mixing}

To reduce the three red-green-blue channels of the color video frames to a one-channel, two-dimensional array for PIV cross-correlation, two of the channels were simply discarded (Figure 3b) from the original image (Figure 3a). Selecting only the red channel data provided a higher bubble-to-background contrast than either the green or blue channels (the color of the pool made the image background brighter in green and blue than in red). The red channel also had higher contrast than the equivalent grayscale image. MATLAB calculates grayscale from rgb using the luminance-weighted average of $0.2989 \mathrm{R}+0.5870 \mathrm{G}+0.1140 \mathrm{~B}$; however, other combinations (including negative weights) were examined here, and none were found to produce better data than the red channel alone. When generalizing this procedure, the best choice for this step is likely to be application-specific.

\subsubsection{Sliding Minimum Subtraction}

The next pre-processing steps isolate the bubbles from the rest of the image by identifying characteristics which the bubbles do not share with the background. The first of these steps removes slow or stationary objects from the background by the subtraction of a temporal sliding minimum. This is a familiar background filter for laboratory PIV as well [30]. The value of every pixel in the series of video frames was reduced by the lowest value of that same pixel in the frames within a specified filter width: here, this was three frames. Pixels with (near) constant values were reduced to (near) zero, whereas the momentary passing of a seed particle/bubble was maintained. Figure $3 \mathrm{c}$ is colored by the values to be subtracted, and Figure $3 \mathrm{~d}$ shows the result of this step: the stationary background and bubble-generating apparatus are removed from the image, and the moving bubbles, refracted sunlight caustics, and the border of the sea lion remain visible.

\subsubsection{Spatial Median Subtraction}

The border of the swimming sea lion and the image of out-of-focus bubbles could be removed by median filter subtraction. A median filter replaced every pixel in an image with the median value from that pixel's neighbors in a specified $\mathrm{m} \times \mathrm{n}$ region: here, $7 \times 7$ pixels. This left large, smooth areas of an image unaffected, but replaced objects much smaller than the filter area with the local background values. This was the opposite of the desired effect here; therefore, that median filtered result was subtracted from the input data to remove the large objects and leave the smaller ones. Figure $3 e$ is colored by the values to be subtracted, and Figure $3 \mathrm{f}$ shows that the bubbles remained, but most of the background has been eliminated. 

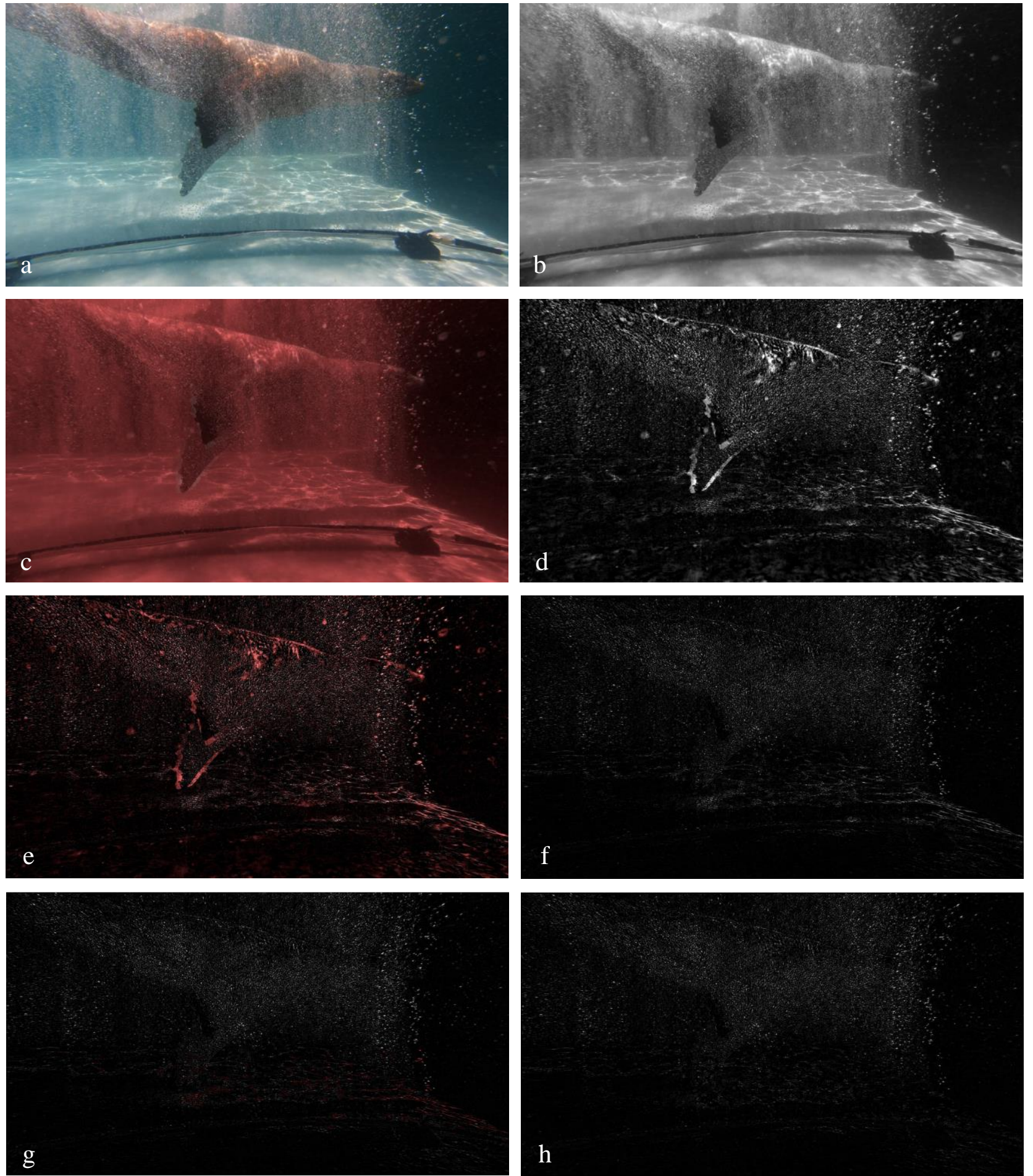

Figure 3. This figure shows the steps in image preprocessing and the changes that each step yields: (a) the raw data, (b) with only the red channel selected, (c) with the sliding minimum subtracted, (d) after a spatial median subtraction, (e) after a heuristic refinement, and, finally, (f) the preprocessed data that was used for velocity field calculations (g) is colored by the values to be subtracted (h) shows the result. This process is detailed in Section 2.2 and is necessary to calculate velocity fields from the in situ data.

\subsubsection{Heuristic Refinement}

The pre-processing steps removed background objects that were slow (by temporal minimum subtraction) and large (by spatial median subtraction), but the sunlight caustics 
on the pool floor were neither, and survived the process. In other similar experiments, this might be avoided by angling the camera to avoid any overlap of the region of interest and the pool floor, but that was not practical here. Instead, it was noted that the nature of the refraction patterns caused them to be connected in intricate patterns; thus, they were isolated from the bubbles by masking regions of the filtered image in which the illuminated pixels belonged to a connected area exceeding a size threshold: here, 50 or more connected pixels brighter than the background intensity. Again, Figure $3 g$ is colored by the values to be subtracted and Figure $3 \mathrm{~h}$ shows the result. The correlation-ready data are shown; only the bubbles remain visible.

\subsection{Two-Dimensional PIV}

The grayscale images showing only in-focus bubbles are ready for two-dimensional PIV calculation through sequential cross-correlation. The velocity calculations in this work were carried out in LaVision's DaVis software. Correlation was performed iteratively with two passes using decreasing window size; velocities were first estimated by correlations between aligned $64 \times 64$-pixel interrogation windows, and then refined by correlation between $32 \times 32$-pixel windows offset by the smoothed initial estimates. In both passes, a $50 \%$ window overlap was used. Vectors with magnitudes greater than two standard deviations away from the mean of their $5 \times 5$ vector neighborhood were considered outliers and replaced by the median of that region. This selective replacement method leaves all non-outlier vectors unchanged. The results are shown in Figure 4b, drawn over the original image (Figure 4a) and colored by the velocity magnitude.
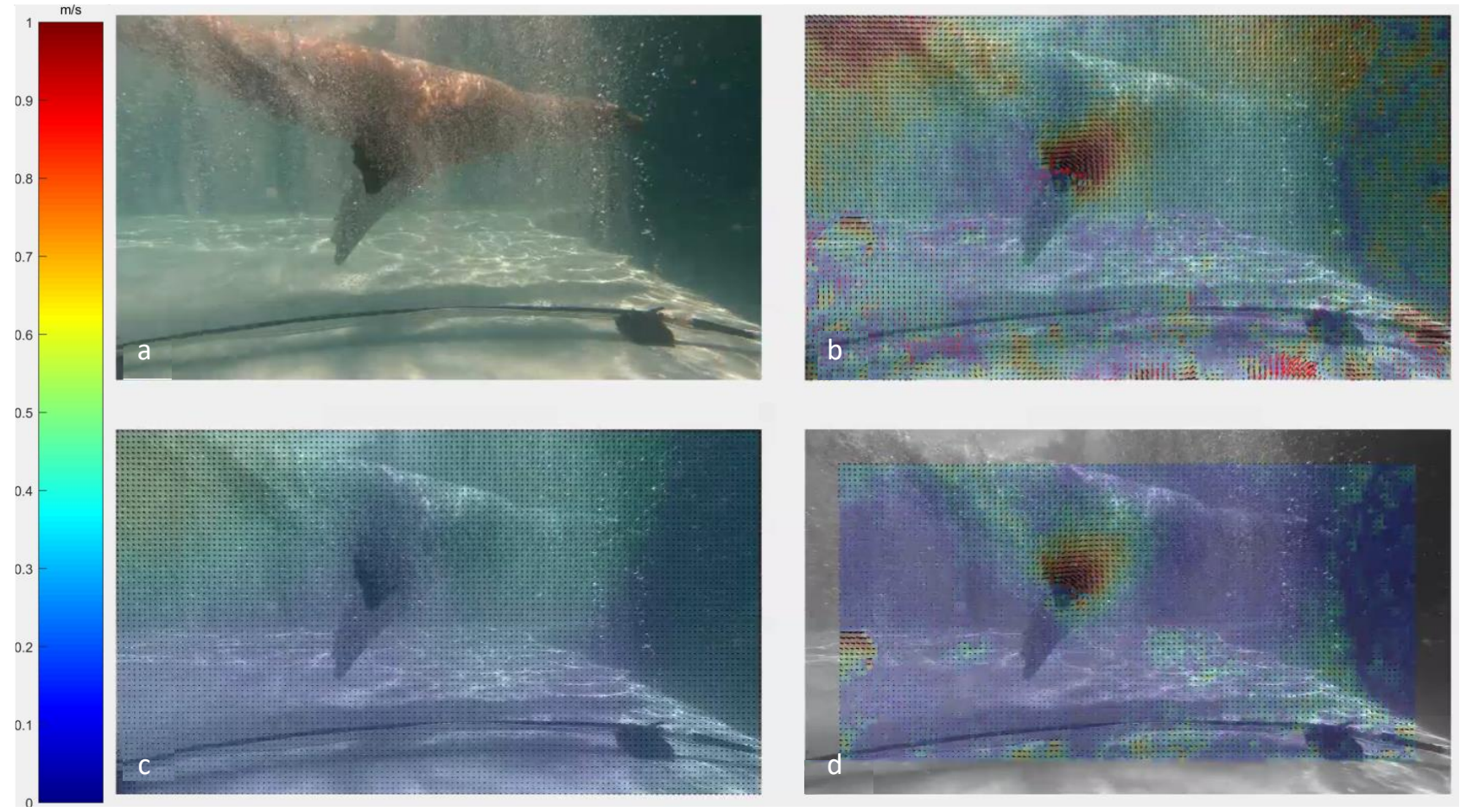

Figure 4. The buoyant rise of the bubble tracer particles must be removed from the processed data. This figure shows the raw data (a), the processed data without accounting for the bubble rise (b), the bubble velocity (c), and the velocity field with the bubble's rise velocity removed (d). This process is detailed in Section 2.3.

However, the velocities calculated by PIV corresponded to the bubbles, not directly to the water. The bubbles are buoyant, unlike typical PIV particles; therefore, the bubble and water velocities are not identical. The bubbles observed in this study rise at a velocity of approximately $0.3 \mathrm{~m} / \mathrm{s}$. The sea lions moved at approximately one body length per second 
(this varied by individual and run, but was in the order of $1.5 \mathrm{~m} / \mathrm{s}$ ). The fluid velocities measured near the sea lions' foreflippers were approximately $1 \mathrm{~m} / \mathrm{s}$ (this is discussed in Section 3.1). Moreover, the limits on environmental control in this and any similar experiment mean that the water is not as quiescent as would be desirable for measuring the flow induced by swimming sea lions. This can be addressed in a similar manner to the image preprocessing: the background flow can be isolated and removed by the separation of time and length scales. The background velocity of the bubbles-caused by both their buoyancy and by the sloshing of the water in the pool-varies over larger length and time scales compared to the flow caused by the faster, smaller sea lion. This was performed in a single step by calculating a three-dimensional median subtraction filter across the two spatial dimensions of the images and the time dimension between consecutive frames; here, regions of $29 \times 29$ vectors across 7 frames. The background velocity calculated in this way is shown in Figure 3c. This background velocity was removed from the vectors calculated in DaVis (Figure $3 \mathrm{~b}$ ) and the final result of the flow induced by the sea lion was determined (Figure 3d).

\section{Results}

In total, 36 instances of sea lion propulsive clap were captured and processed. This required 173 measurements across two sessions with three individual sea lions (the sea lions only performed trained behaviors while being fed, and data could only be collected when the sun was overhead). Only 58 of the videos included the propulsive clap within the measurement region. Several of those videos were unable to be processed due to changing illuminations or increasingly turbid water.

\subsection{Velocity Fields}

A representative velocity field was observed throughout the propulsive stroke of the sea lion (all other instances are available in the upon direct request from the authors). Figure 5 shows the velocity field at six equally spaced times throughout the propulsive stroke. As the animal approached the bubble sheet, before reaching it, the velocity field (right side of panel a) predictably showed little to no flow. Notable, despite our best efforts, these types of field data will always be noisy compared to laboratory experiments. Fortunately, the noise-in this case, caused by the motion of the sun refraction on the bottom of the pool-is typically easily distinguished from the signal we are measuring.

As the animal approached (top left of panel a), the area directly upstream of the foreflipper was disturbed (seen in the upper center of Figure 5a). As the flipper swept through the measurement plane (panels b and c), the highest velocity values were seen at the tip of the foreflipper and on the upper, suction side of this propulsive surface. In panel c, it is clear that the higher velocity region was located on the suction side of the flipper, being accelerated behind the fast-moving surface.

As the propulsive stroke ended (panel d) and the animal glided behind the measurement plane (panels e and f), the velocity field remained disturbed where the sea lion accelerated the flow behind it. In the measurement plane, we observed a disturbance to the velocity field caused by this structure diagonally from the upper left corner to the center of Figure 5e. A wake structure can be seen in the raw images in Figure 6. The top panel (Figure 6a) is the raw data used in Figures 5e and 7e. The structure is highlighted by the red circle. The lower panel (Figure $6 \mathrm{~b}$ ) is from a different run. The animal was in a slightly different plane, resulting in this structure being more clearly visible. The strength of this structure decreased (panel f) as the animal moved further downstream. 

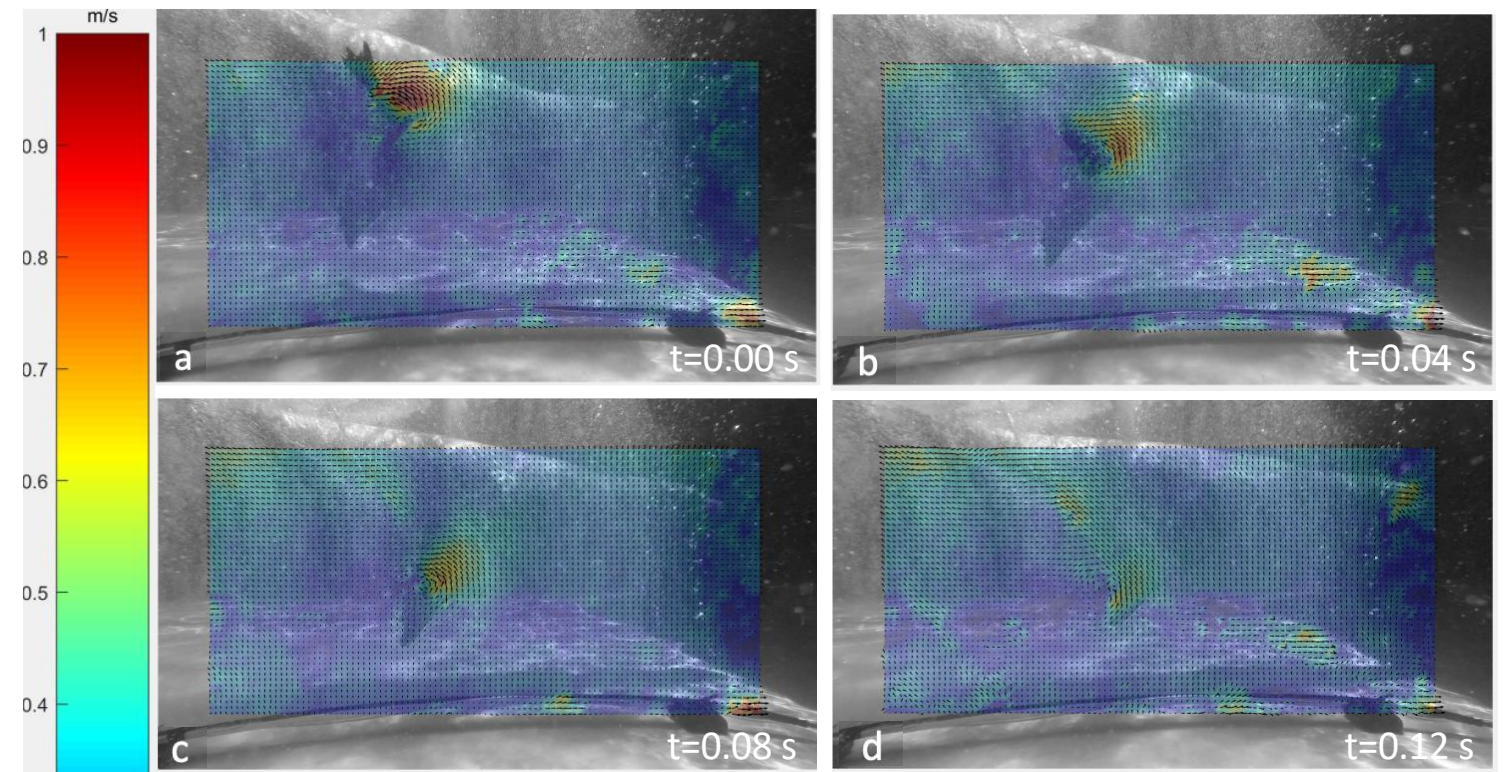

0.3
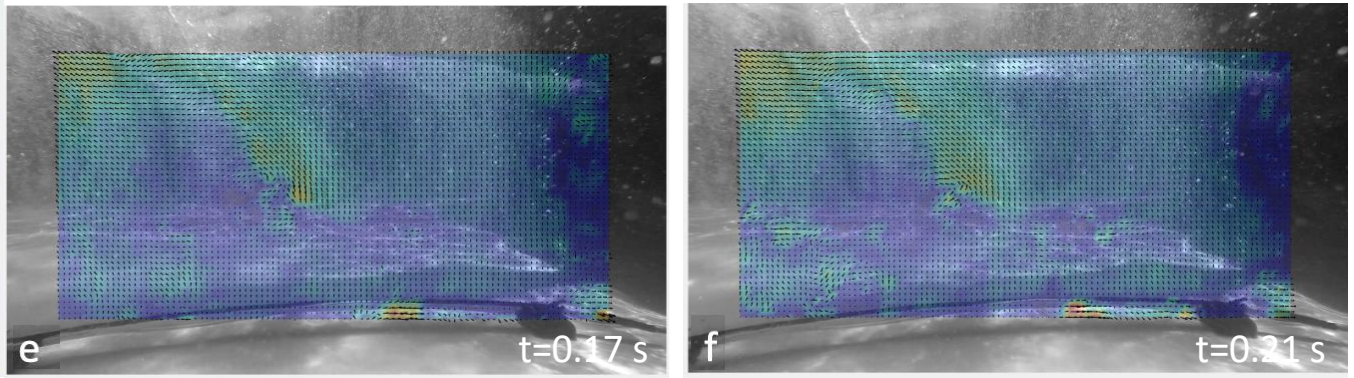

Figure 5. The velocity fields measured with bubble PIV of a swimming sea lion were plotted at 6 instances in time as the sea lion completed a propulsive stroke in the plane of the bubble sheet: (a) as the animal enters the sheet, (b-d) while the propulsive stroke is actively being completed, and $(\mathbf{e}, \mathbf{f})$ while the sea lion glides through the measurement plane. Images show raw data (underlay) with velocity magnitude (colormap) and velocity vectors.

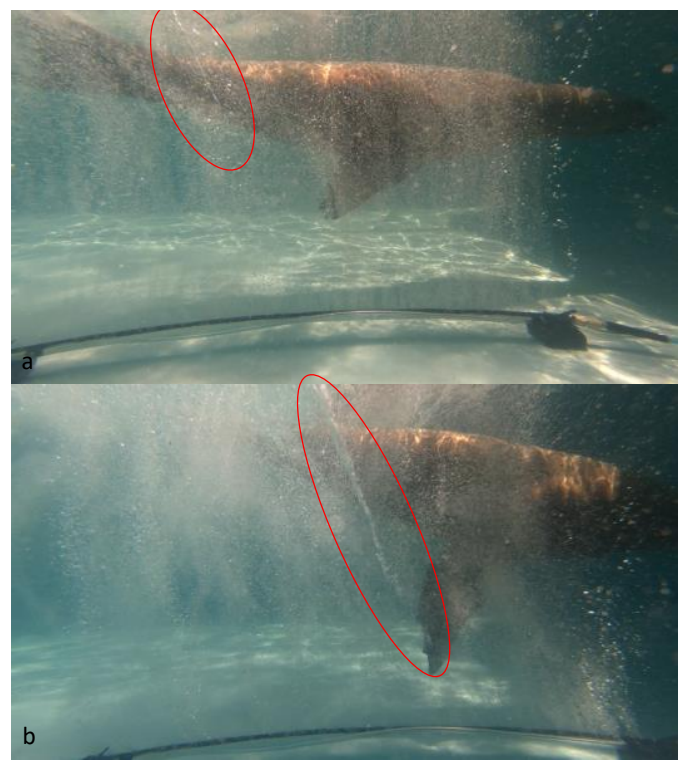

Figure 6. The top panel (a) shows the raw data used to produce Figures $5 \mathrm{e}$ and $7 \mathrm{e}$, and the bottom panel (b) is a similar phase in another data run. Both show a trailing vortex structure (red circle) resulting from the sea lion's propulsive stroke. 

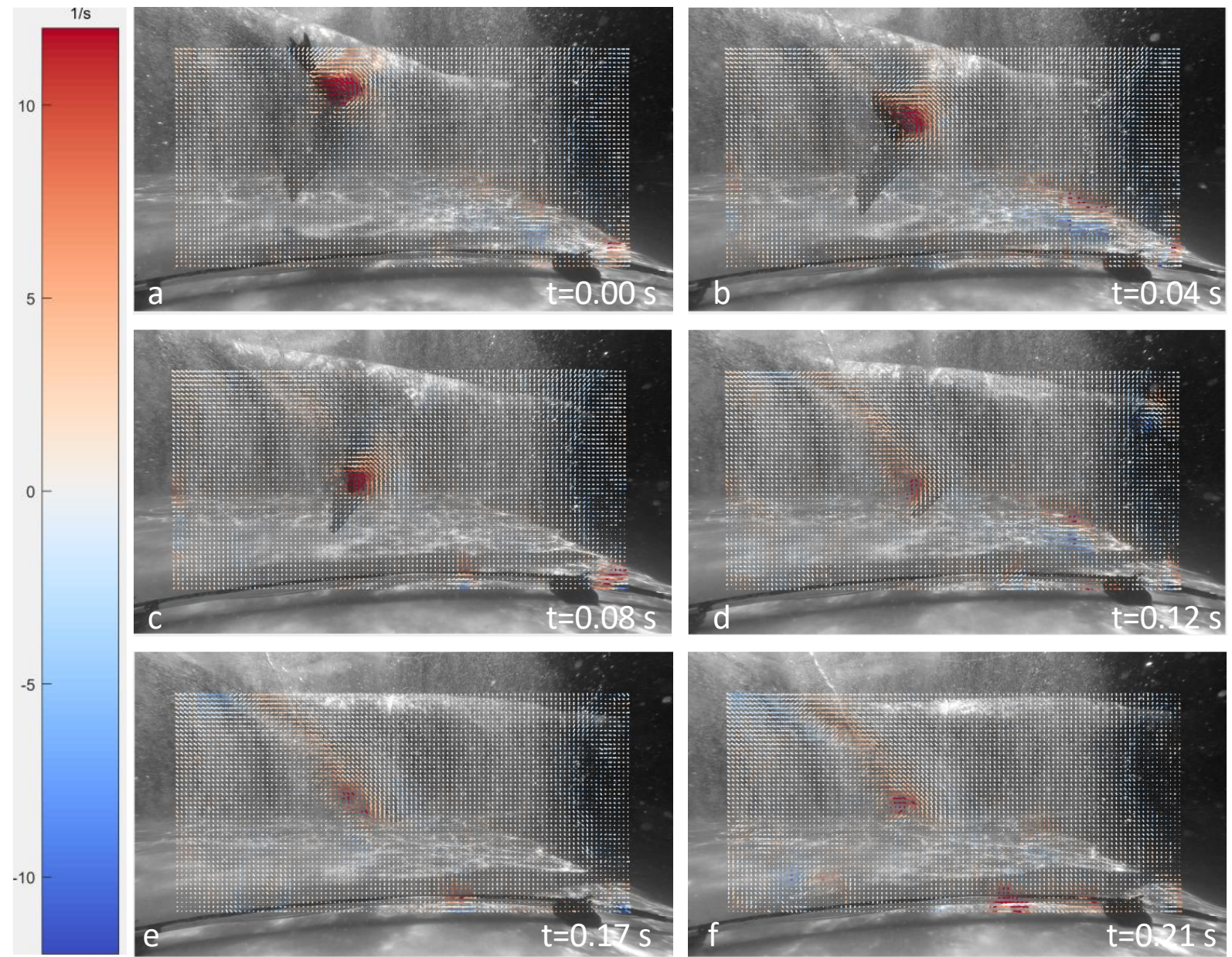

Figure 7. The vorticity fields calculated from the bubble PIV (shown in Figure 5) of a swimming sea lion were plotted at 6 instances as the sea lion completed a propulsive stroke in the plane of the bubble sheet: (a) as the animal enters the sheet, $(\mathbf{b}-\mathbf{d})$ while the propulsive stroke is actively being completed, and $(\mathbf{e}, \mathbf{f})$ while the sea lion glides through the measurement plane. Images show raw data (underlay) with vorticity magnitude (colormap) and velocity vectors.

\subsection{Vorticity Fields}

The velocity fields measured and presented above were used to calculate the vorticity field of the free-swimming sea lion. Figure 6 presents the vorticity fields which corresponded to the velocity fields in Figure 5 (all others are available upon request). A strong region of positive vorticity is seen forming on the leading/suction edge of the foreflipper as it began and executes the propulsive stroke (Figure 7a-c). Positive vorticity corresponds to counterclockwise rotation of the fluid. As the stroke completed and the animal glided out of the measurement field, the vortex structure detached from the flipper. This persisted downstream of the animal until it was out of frame (bottom row of Figure 7). The structure was three-dimensional in nature; thus, the results displayed in Figure 7 show just those visible in the measurement plane. The elongated region of positive vorticity (red) with a weaker negative vorticity signal around it (Figure 7, bottom leftmost panel) was, in reality, a vortex tube that stretched from the animal's foreflipper to the water's surface. The structure is visible in the raw data images shown in Figure 6.

\section{Discussion}

This study highlights the difficulty of working in the field at full-scale with living animals, particularly large animals. Although the methods and results do provide additional tools for these studies, they are still limited, compared with the quantitative data obtained 
in the laboratory with complete or partial models. However, they can provide a benchmark for model systems to determine whether those models capture the relevant flow physics accurately. The results presented above were compared to velocity and vorticity fields measured on a robotic sea lion foreflipper, as presented by Kashi et al. [23]

The main qualitative result from Kashi et al. is that there is a large structure which forms on the suction side (away from the body) of the robotic flipper [23]. This structure's strength increased as the flipper approaches the "body", modelled by a flat plate mounted perpendicularly to the robotic flipper. That structure then convected downstream (in the robotic experiments, the robotic flipper was fixed, as opposed to the moving animal). This is the same general flowfield that was measured in situ using bubble PIV, as shown in Figures 4 and 6 . In both cases, a strong fluid structure can be seen on the suction side of the flipper (real or robotic). Although the robotic foreflipper used by Kashi et al. lacked much of the complexity of the full-scale animals, especially the three dimensionality, it provided a platform for much more detailed and in-depth exploration. By combining the in situ results presented here and laboratory-based models, a more complete picture of sea lion propulsion has emerged.

\section{Conclusions}

Particle image velocimetry represents an exceptional tool for measuring the velocity fields of many physical flows. However, there are still cases where traditional PIV methods cannot be used. This study extends its usefulness to flows that cannot be adapted to the laboratory setting, or where laser light cannot be used for practical or safety reasons. Additionally, it provides the first in situ measurements of the flowfield generated by a freely swimming sea lion. It shows the strong flow features produced by the propulsive stroke of the sea lion. Finally, it provides a benchmark for laboratory experiments of robotic sea lion foreflipper platforms.

Author Contributions: F.E.F. and M.C.L. conceptualized and supervised the project. All authors, except for M.C.L., collected that data at the SLEWTHS field site in Moss Landing, CA. G.P. processed the data and developed the results. G.P., M.C.L. and F.E.F. prepared the manuscript and figures. All authors have read and agreed to the published version of the manuscript.

Funding: Funding for this study was provided by the National Science Foundation (CBET-1604876), and the Office of Naval Research (N0014-17-1-2448 to MCL from Robert Brizzolara and N0014-17-2312 to M.C.L. (PI) and F.E.F. (co-PI) from Thomas McKenna).

Institutional Review Board Statement: The experiments on the sea lions was approved by the West Chester University Institutional Animal Care and Use Committee (IACUC) under protocol number 2016R3.

Data Availability Statement: All raw and processed data reported in this manuscript and those of runs which could not be included for length purposes are available upon request to the authors.

Acknowledgments: This study could not have been conducted without the excellent team at the Science and Learning With the Help of Sea Lions (SLEWTHS) center in Moss Landing, Ca. Special thanks are given to SLEWTHS director Jenifer Zeligs and trainer Stafani Skrovan. Data were collected with three sea lions: Cali, Ariel, and Nemo.

Conflicts of Interest: The authors declare no conflict of interest.

\section{References}

1. Drucker, E.G.; Lauder, G.V. Locomotor forces on a swimming fish: Three-dimensional vortex wake dynamics quantified using digital particle image velocimetry. J. Exp. Biol. 1999, 202, 2393-2412. [CrossRef]

2. Lauder, G.V.; Drucker, E.G. Forces, fishes, and fluids: Hydrodynamic mechanisms of aquatic locomotion. Physiology 2002, 17, 235-240. [CrossRef]

3. Tytell, E.D.; Lauder, G.V. The hydrodynamics of eel swimming: I. Wake structure. J. Exp. Biol. 2004, 207, 1825-1841. [CrossRef]

4. Tytell, E.D. The hydrodynamics of eel swimming II. Effect of swimming speed. J. Exp. Biol. 2004, 207, 3265-3279. [CrossRef]

5. Siddiqui, M.H.K. Velocity measurements around a freely swimming fish using PIV. Meas. Sci. Technol. 2006, 18, 96-105. [CrossRef] 
6. Dabiri, J.O.; Colin, S.P.; Gemmell, B.J.; Lucas, K.N.; Leftwich, M.C.; Costello, J.H. Jellyfish and Fish Solve the Challenges of Turning Dynamics Similarly to Achieve High Maneuverability. Fluids 2020, 5, 106. [CrossRef]

7. Mendelson, L.; Techet, A.H. Quantitative wake analysis of a freely swimming fish using 3D synthetic aperture PIV. Exp. Fluids 2015, 56, 135. [CrossRef]

8. Mendelson, L.; Techet, A.H. Multi-camera volumetric PIV for the study of jumping fish. Exp. Fluids 2017, 59, 10. [CrossRef]

9. Triantafyllou, M.S.; Yue, D.K.P. Hydrodynamics of Fishlike Swimming. Annu. Rev. Fluid Mech. 2000, 32, 33-53. [CrossRef]

10. Wu, T.Y. Fish Swimming and Bird/Insect Flight. Annu. Rev. Fluid Mech. 2011, 43, 25-58. [CrossRef]

11. Lauder, G.V. Fish Locomotion: Recent Advances and New Directions. Annu. Rev. Mar. Sci. 2015, 7, 521-545. [CrossRef]

12. Costello, J.H.; Colin, S.P.; Dabiri, J.O.; Gemmell, B.J.; Lucas, K.N.; Sutherland, K.R. The Hydrodynamics of Jellyfish Swimming. Annu. Rev. Mar. Sci. 2021, 13, 375-396. [CrossRef] [PubMed]

13. Katija, K.; Dabiri, J.O. In situ field measurements of aquatic animal-fluid interactions using a Self-Contained Underwater Velocimetry Apparatus (SCUVA). Limnol. Oceanogr. Methods 2008, 6, 162-171. [CrossRef]

14. Dabiri, J.O.; Gharib, M.; Colin, S.P.; Costello, J.H. Vortex motion in the ocean: In situ visualization of jellyfish swimming and feeding flows. Phys. Fluids 2005, 17, 091108. [CrossRef]

15. Troutman, V.A.; Dabiri, J.O. Single-camera three-dimensional tracking of natural particulate and zooplankton. Meas. Sci. Technol. 2018, 29, 075401. [CrossRef]

16. Katija, K.; Sherlock, R.E.; Sherman, A.D.; Robison, B.H. New technology reveals the role of giant larvaceans in oceanic carbon cycling. Sci. Adv. 2017, 3, e1602374. [CrossRef] [PubMed]

17. Tolkoff, S.W. Robotics and Power Measurements of the RoboTuna. Ph.D. Thesis, Massachusetts Institute of Technology, Cambridge, MA, USA, 1999.

18. Hultmark, M.; Leftwich, M.; Smits, A.J. Flowfield measurements in the wake of a robotic lamprey. Exp. Fluids 2007, 43, 683-690. [CrossRef] [PubMed]

19. Ijspeert, A.J.; Crespi, A.; Ryczko, D.; Cabelguen, J.-M. From Swimming to Walking with a Salamander Robot Driven by a Spinal Cord Model. Science 2007, 315, 1416-1420. [CrossRef]

20. Leftwich, M.C.; Smits, A.J. Thrust production by a mechanical swimming lamprey. Exp. Fluids 2010, 50, 1349-1355. [CrossRef]

21. Tangorra, J.L.; Lauder, G.V.; Hunter, I.W.; Mittal, R.; Madden, P.G.A.; Bozkurttas, M. The effect of fin ray flexural rigidity on the propulsive forces generated by a biorobotic fish pectoral fin. J. Exp. Biol. 2010, 213, 4043-4054. [CrossRef]

22. Esposito, C.J.; Tangorra, J.L.; Flammang, B.; Lauder, G.V. A robotic fish caudal fin: Effects of stiffness and motor program on locomotor performance. J. Exp. Biol. 2012, 215, 56-67. [CrossRef]

23. Kashi, E.; Kulkarni, A.; Perrotta, G.M.; Leftwich, M.C. Flowfields produced by a robotic sea lion foreflipper starting from rest. Bioinspir. Biomim. 2020, 15, 035002. [CrossRef]

24. Fish, F.E.; Legac, P.; Williams, T.M.; Wei, T. Measurement of hydrodynamic force generation by swimming dolphins using bubble DPIV. J. Exp. Biol. 2014, 217, 252-260. [CrossRef]

25. Fish, F.E.; Williams, T.M.; Sherman, E.; Moon, Y.E.; Wu, V.; Wei, T. Experimental Measurement of Dolphin Thrust Generated during a Tail Stand Using DPIV. Fluids 2018, 3, 33. [CrossRef]

26. Ryu, Y.; Chang, K.-A.; Lim, H.-J. Use of bubble image velocimetry for measurement of plunging wave impinging on structure and associated greenwater. Meas. Sci. Technol. 2005, 16, 1945-1953. [CrossRef]

27. Friedman, C.; Leftwich, M.C. The kinematics of the California sea lion foreflipper during forward swimming. Bioinspir. Biomim. 2014, 9, 046010. [CrossRef] [PubMed]

28. Godfrey, S.J. Additional observations of subaqueous locomotion in the California Sea Lion (Zalophus californianus). Aquat. Mamm. $1985,11,53-57$.

29. Feldkamp, S.D. Foreflipper propulsion in the California sea lion, Zalophus californianus. J. Zool. 1987, 212, 43-57. [CrossRef]

30. Adrian, R.J.; Westerweel, J. Particle Image Velocimetry (No. 30); Cambridge University Press: Cambridge, UK, 2011. 